\title{
Interband Optical Properties of Concentric Type-I Nanorings in a Normal Magnetic Field
}

\author{
V. Arsoski ${ }^{a, *}$, M. TAdiĆ $^{a}$ And F.M. PeEters ${ }^{b}$ \\ ${ }^{a}$ Faculty of Electrical Engineering, University of Belgrade, P.O. Box 3554, 11120 Belgrade, Serbia \\ ${ }^{b}$ Department of Physics, University of Antwerp, Groenenborgerlaan 171, B-2020 Antwerp, Belgium \\ Two concentric two-dimensional GaAs/(Al,Ga)As nanorings in a normal magnetic field are theoretically \\ studied. The single-band effective mass approximation is adopted for both the electron and the hole states, and \\ the analytical solutions are given. We find that the electronic single particle states are arranged in pairs, which \\ exhibit anticrossings and the orbital momentum transitions in the energy spectrum when magnetic field increases. \\ Their period is essentially determined by the radius of the outer ring. The oscillator strength for interband \\ transitions is strongly reduced close to each anticrossing. We show that an optical excitonic Aharonov-Bohm \\ effect may occur in concentric nanorings.
}

PACS numbers: 73.21.La, 78.67.Hc

\section{Introduction}

The shape of a nanodot depends on the growth method. An interesting example is the one in the shape of a ring [1-3], which shows oscillations in the electron and hole ground state energy with varying magnetic field [4]. The oscillations are due to the Aharonov-Bohm effect, which is the result of double connected topology of the ring and wave nature of the charge carriers [5]. Different spectroscopic techniques confirm such orbital momentum transitions in nanorings [4], which give rise to oscillations in the ground electron energy. These nanorings have typical lateral dimensions of the order of $10 \mathrm{~nm}$, while they are only a few $\mathrm{nm}$ high [6]. Their fabrication allows the exploration of topological effects even for neutral systems, such as excitons [7]. The excitonic and optical excitonic Aharonov-Bohm effects have been recently explored both theoretically and experimentally $[8,9]$. The former effect is demonstrated by oscillations of the ground exciton state as a function of the magnetic field [7], whereas the latter effect is an optical property of the exciton [10]: the transitions between the single particle states of different orbital momenta may turn from bright to dark. The optical excitonic Aharonov-Bohm effect was predicted for two concentric one-dimensional (1D) rings [10].

There has been a growing interest in systems of multiple quantum rings, including concentric rings [11-14], vertically stacked rings [3, 15], and ring arrays [16]. Double concentric nanorings have been recently fabricated by the modified droplet epitaxy technique [11]. These rings are made of GaAs embedded in a $(\mathrm{Al}, \mathrm{Ga}) \mathrm{As}$ ma-

\footnotetext{
* corresponding author; e-mail: arsosk@etf.rs
}

trix, and show negligible deviations from axial symmetry $[11,12]$. Also, their size and shape in the realized ensembles exhibit high uniformity, which makes them suitable for photonic applications [13]. A recent theoretical study of two electrons confined in such concentric rings indicated that the periodicity of the Aharonov-Bohm oscillations strongly depends on the width of the barrier between the rings [14].

In this paper, we explore the electronic structure and the interband optical properties of the electron and the hole in two concentric GaAs/( $\mathrm{Al}, \mathrm{Ga}) \mathrm{As}$ nanorings. We adopt the effective-mass theory and the adiabatic approximation to compute the single particle states. The adiabatic approximation is suitable because the rings height is much smaller than their lateral dimensions. The Schrödinger equation is solved analytically. A normal magnetic field through the rings is varied in order to explore how the energy levels and the oscillator strength are affected by the coupling between two rings. Two rings are shown schematically along with the confinement potential profiles in the conduction and the valence band in Fig. 1. The inner radii of two rings are denoted by $\rho_{1}$ and $\rho_{3}$ while the outer radii are denoted by $\rho_{2}$ and $\rho_{4}$. The confining potential assumed in our model has the rectangular shape. Such a choice differs from our previous work [14], where the parabolic-like potential was assumed.

The paper is organized as follows. The theory of the electron and hole states is presented in Sect. 2. The numerical results are presented and discussed in Sect. 3.

\section{Theory}

We consider two-dimensional concentric rings, located in the $x y$ plane. A magnetic field is applied along the 


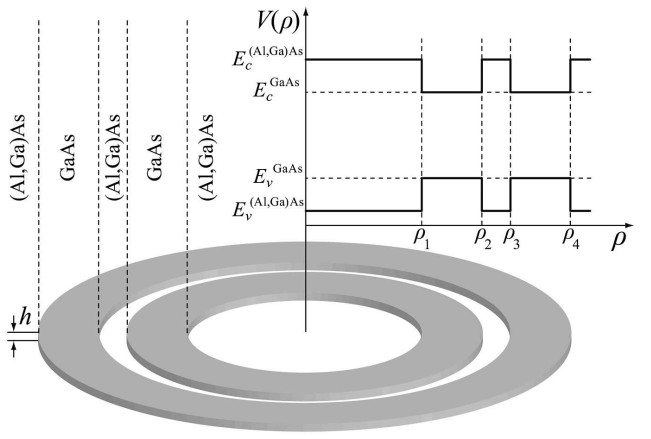

Fig. 1. A schematic view of two concentric quantum rings, and the confinement potentials in the conduction and the valence band.

$z$ direction and cylindrical coordinates are used. The single-band effective mass Hamiltonian in the adiabatic approximation is used for both the electron and hole states. Even though much more elaborate models can be employed [17], the small height of the fabricated rings brings about large splitting between the heavy and light hole levels, and therefore mixing between them is reduced. Furthermore, the large band gap of GaAs, which is assumed to be a material of the ring, justifies use of the single-band approximation in the conduction band. The two dimensional Hamiltonian, for in plane motion, is given by

$$
H=(\boldsymbol{P}-q \boldsymbol{A}) \frac{1}{2 m^{*}}(\boldsymbol{P}-q \boldsymbol{A})+V(\rho) .
$$

Here $m^{*}$ denotes the position dependent effective mass of the particle, $\boldsymbol{A}$ is the magnetic vector potential, $q$ is the elementary charge $(q=-e$ for the electron and $q=+e$ for the heavy and light holes), and $V(\rho)$ is the confinement potential in the radial direction. For a uniform magnetic field, the vector potential can be written as $\boldsymbol{A}=\boldsymbol{B} \times \boldsymbol{r} / 2$, which leads to the Schrödinger equation:

$$
\begin{gathered}
-\frac{\hbar^{2}}{2}\left(\frac{1}{\rho} \frac{\partial}{\partial \rho}\left(\frac{\rho}{m^{*}} \frac{\partial \Psi}{\partial \rho}\right)+\frac{1}{m^{*} \rho^{2}} \frac{\partial^{2} \Psi}{\partial \phi^{2}}\right)+\frac{\mathrm{i} \hbar q B}{2 m^{*}} \frac{\partial \Psi}{\partial \phi} \\
+\frac{m^{*}\left(q B / m^{*}\right)^{2} \rho^{2}}{8} \Psi+[V(\rho)-E] \Psi=0
\end{gathered}
$$

The axial symmetry of the Hamiltonian allows for the factorization of the wave function,

$$
\Psi_{n, l}(\rho, \phi)=\frac{\mathrm{e}^{\mathrm{i} l \phi}}{\sqrt{2 \pi}} R_{n, l}(\rho), \quad l=0, \pm 1, \pm 2, \pm 3, \ldots,(3)
$$

and the differential equation for the radial part of the wave function is easily derived,

$$
\begin{array}{r}
-\frac{\hbar^{2}}{2}\left(\frac{\partial}{\partial \rho}\left(\frac{1}{m^{*}} \frac{\partial R_{n, l}}{\partial \rho}\right)+\frac{1}{m^{*}} \frac{1}{\rho} \frac{\partial R_{n, l}}{\partial \rho}-\frac{l^{2}}{\rho^{2}} R_{n, l}\right) \\
\pm \frac{\hbar l \omega_{\mathrm{c}}}{2} R_{n, l}+\frac{m^{*} \omega_{\mathrm{c}}^{2} \rho^{2}}{8} R_{n, l}+[V(\rho)-E] R_{n, l}=0
\end{array}
$$

where $\omega_{\mathrm{c}}=\left|q B / m^{*}\right|$ is the position dependent cyclotron frequency. The upper and the lower sign are for the elec- tron and the hole, respectively. If we use the dimensionless variable $\xi=e B \rho^{2} /(2 \hbar)$, the differential equation for the radial wave function becomes

$$
\begin{aligned}
& \xi R_{n, l}^{\prime \prime}(\xi)+R_{n, l}^{\prime}(\xi) \\
& \quad+\left(\frac{E-V(\xi)}{\omega_{\mathrm{c}}^{\beta}}-\frac{l^{2}}{4 \xi} \mp \frac{l}{2}-\frac{\xi}{4}\right) R_{n, l}(\xi)=0
\end{aligned}
$$

in the well $(\beta=w)$ and the barrier $(\beta=b)$. Equation (5) admits solutions of the form

$$
R_{n, l}(\xi)=\mathrm{e}^{-\xi / 2} \xi^{|l| / 2} f_{n, l}(\xi) .
$$

The function $f_{n, l}(\xi)$ satisfy the equation

$$
\xi f_{n, l}^{\prime \prime}(\xi)+(b-\xi) f_{n, l}^{\prime}(\xi)-a f_{n, l}(\xi)=0,
$$

where $a_{\beta}=|l| / 2+1 / 2 \pm l / 2+\left(V_{\beta}-E\right) /\left(\hbar \omega_{\mathrm{c}}^{\beta}\right)$, and $b=|l|+1$. The general solution of this equation may be expressed as a linear combination of the confluent hypergeometric functions of the first kind and the second kind, $M_{n, l}$ and $U_{n, l}$, respectively:

$$
f_{n, l}(\xi)=A M_{n, l}(a, b, \xi)+B U_{n, l}(a, b, \xi) .
$$

Applying the boundary conditions

$$
\begin{aligned}
& R_{n, l}\left(\rho_{i}^{-}\right)=R_{n, l}\left(\rho_{i}^{+}\right), \\
& \left.\frac{1}{m^{*}(\rho)} \frac{\mathrm{d} R_{n, l}}{\mathrm{~d} \rho}\right|_{\rho=\rho_{i}^{-}}=\left.\frac{1}{m^{*}(\rho)} \frac{\mathrm{d} R_{n, l}}{\mathrm{~d} \rho}\right|_{\rho=\rho_{i}^{+}},
\end{aligned}
$$

at the interfaces between the rings and the matrix, a set of eight algebaric equations is obtained, from which the eigenenergies are extracted.

The oscillator strength for interband transitions is given by [18]:

$$
f=\frac{2}{m_{0} E_{\mathrm{vc}}}\left|M_{\mathrm{cv}}\right|^{2},
$$

where $m_{0}$ denotes the free electron mass, $E_{\mathrm{vc}}=\left|E_{\mathrm{v}}^{\mathrm{GaAs}}\right|+$ $E_{\mathrm{c}}^{\mathrm{GaAs}}+E_{g}^{\mathrm{GaAs}}$, and $M_{\mathrm{cv}}$ is the transition matrix element between the states in the conduction and the valence band, which is given by

$$
\begin{aligned}
& M_{\mathrm{cv}}=\frac{m_{0} P}{\hbar} \delta_{l_{\mathrm{c} j},-l_{\mathrm{v} i}} \\
& \quad \times \int_{\rho=0}^{\infty} \Psi_{\mathrm{c} j} \Psi_{\mathrm{v} i} \rho d \rho \int_{z=-\infty}^{+\infty} Z_{\mathrm{c} j} Z_{\mathrm{v} i} \mathrm{~d} z,
\end{aligned}
$$

where $P$ is the Kane interband matrix element and $Z_{\text {c } j(v i)}$ are the $z$-dependent wavefunctions of the ground electron and hole states. The later are extracted from the $z$-dependent Schrödinger equation, for the rectangular (hard-wall) potential. Such an adiabatic (single-sublevel) approximation is justified by small height of the fabricated rings.

\section{Results and discussion}

The energies of the band extrema in GaAs are taken as reference levels. The effective mass of the electron in GaAs is $0.067 m_{0}$, whereas the heavy and light hole effective masses are $0.45 m_{0}$ and $0.082 m_{0}$, respectively [19]. The effective masses in the $\mathrm{Al}_{x} \mathrm{Ga}_{1-x} \mathrm{As}$ alloy are computed as $(0.067+0.083 x) m_{0},(0.45+0.2 x) m_{0}$, and $(0.082+0.068 x) m_{0}$ for the electron, the heavy hole, 
and the light hole, respectively. The conduction band offset amounts to $0.7482 x \mathrm{eV}$, while the valence band offset is $0.4988 x \mathrm{eV}$. For the mole fraction we assume $x=0.3$. In our calculations, the inner ring has the inner and outer radii of $\rho_{1}=20 \mathrm{~nm}$ and $\rho_{2}=30 \mathrm{~nm}$, respectively, while the radii of the outer ring are $\rho_{3}=35 \mathrm{~nm}$ and $\rho_{4}=45 \mathrm{~nm}$. We assume that the rings are $h=4 \mathrm{~nm}$ high.

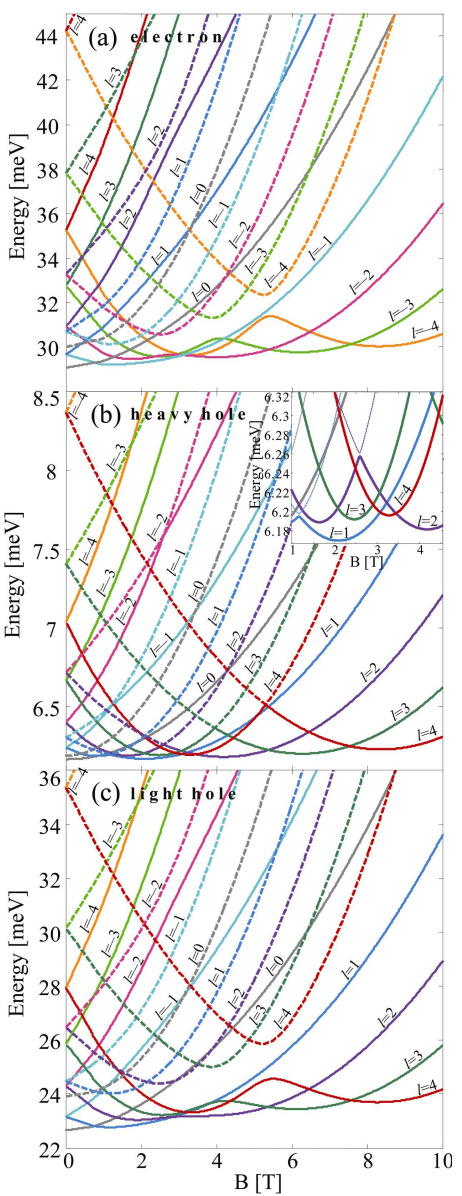

Fig. 2. Energy levels in (a) the conduction, (b) the heavy hole and (c) the light hole band for $l$ in the range $[-4,4]$ as function of a normal magnetic field. The $\mathrm{L}$ states are shown by the solid lines, whereas the dashed lines denote the $\mathrm{H}$ states. The spectrum near anticrossings between the heavy hole states is enlarged and shown in inset.

The magnetic field dependence of the electron, heavy hole, and light hole energies for $l$ in the range $[-4,+4]$ are shown in Figs. 2(a)-(c). All states at $B=0$, except the $l=0$ states, are double degenerate, i.e. $E(l)=E(-l)$. Furthermore, for each orbital momentum, except $l=0$, the wave function of the lowest energy state (L state, shown by the solid line) is mainly localized inside the large ring at $B=0$. On the other hand, the higher energy states ( $\mathrm{H}$ states, shown by the dashed lines) for all $l$ (except $l=0$ ) have their wave functions located mainly inside the small ring. Figure 2 shows that the splitting between the $\mathrm{L}$ and $\mathrm{H}$ states at $B=0$ increases with $|l|$. Furthermore, the L states anticross the respective $\mathrm{H}$ states at a certain value of the magnetic field. Interestingly, the anticrossings in both the conduction and the valence band take place at nearly the same value of the magnetic field. Hence, the locations of the anticrossings are determined by the topology (the ring size) rather than the band structure of the constituent semiconductors (the effective masses, the band offsets etc.). These anticrossings shift towards larger $B$ when $l$ increases.

The energy spectra shown in Fig. 2 may be related to the energy spectra of two concentric 1D rings separated by an infinite barrier. The first energy minimum in each L state of a given $l$ corresponds to an integer number of flux quanta threading the $1 \mathrm{D}$ ring whose radius is given by

$$
B_{\min }=\frac{2 \hbar|l|}{q R_{1 \mathrm{D}}^{2}},
$$

where $R_{1 \mathrm{D}}^{2}=\left(\rho_{3}^{2}+\rho_{3} \rho_{4}+\rho_{4}^{2}\right) / 3$ is the Heronian mean. The second minimum is also described by Eq. (13), but $R_{1 \mathrm{D}}$ is computed as $\sqrt{\left(\rho_{1}^{2}+\rho_{1} \rho_{2}+\rho_{2}^{2}\right) / 3}$. All ground states in Figs. 2a-(c) exhibit Aharonov-Bohm oscillations. Their period mainly depends on the dimensions of the outer ring, but the oscillations in the concentric rings are less regular than in the single ring [5]. This might be ascribed to a change of localization of the electron and the hole from the large ring to the small ring when the magnetic field increases, and was recently confirmed experimentally [20].

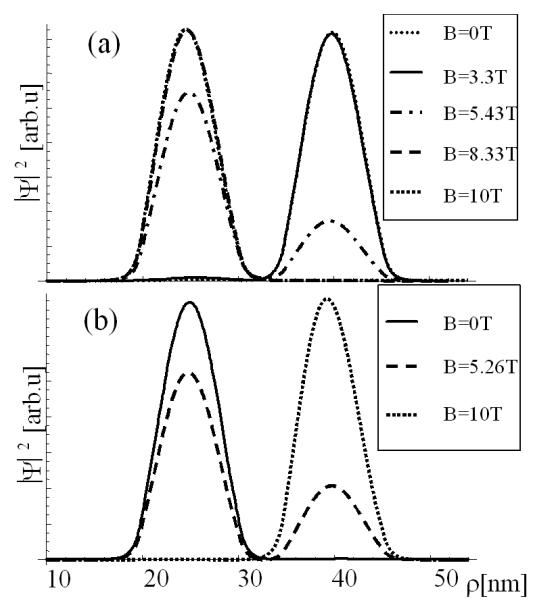

Fig. 3. The probability density of (a) the L and (b) $\mathrm{H}$ state in the conduction band for $l=-4$ and for a few characteristic values of magnetic field in Fig. 2a.

The probability density in the L electron states of the angular momentum $l=-4$ is shown in Fig. 3a, for few characteristic values in Fig. 2a. It shows the electron density for the magnetic field values around the first energy minimum $(3.3 \mathrm{~T})$, the second minimum (8.33 $\mathrm{T})$, the anticrossing $(5.43 \mathrm{~T})$, and for the limiting points of the explored range, 0 and $10 \mathrm{~T}$. When the magnetic field is lower than $3.3 \mathrm{~T}$, the electron is almost fully localized 
in the outer ring. When $B$ increases further, the probability density in the inner ring increases, and at $5.43 \mathrm{~T}$, the probabilities of finding electron in the inner and outer ring are comparable. At the position of the second minimum, the electron in the $\mathrm{L}$ state is almost completely localized inside the inner ring. Further increase of $B$ causes a shift of the maximum of the probability density towards the inner radius of the inner ring. Figure $3 \mathrm{~b}$ shows the probability density in the $\mathrm{H}$ state for $l=-4$ and for a few characteristic points in Fig. 2a.

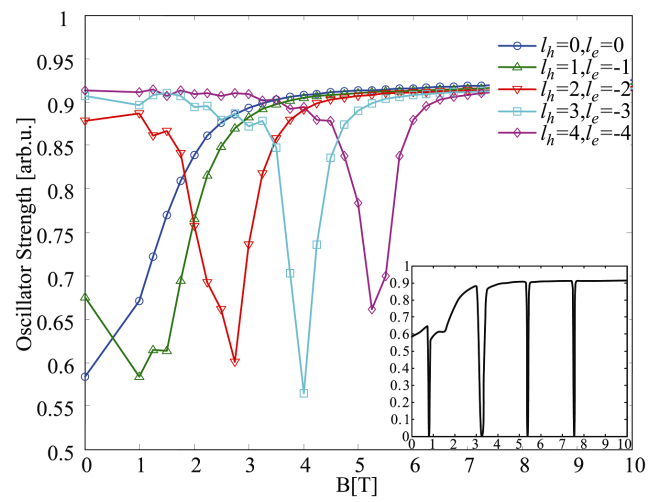

Fig. 4. The oscillator strength for the interband optical transitions between the lowest energy states of the electron and the heavy hole of the opposite orbital momentum $\left(l_{h}=-l_{e}\right)$ as function of a normal magnetic field. Inset shows the oscillator strength for the transitions between the ground states of the electron and the heavy hole.

Figure 4 shows the oscillator strength for interband transitions between the $\mathrm{L}$ states in the conduction band and the heavy hole band. The oscillator strength for each transition shown in Fig. 4 have a local minimum around the anticrossing. The minimum arises from different localizations of the wave functions in the conduction and valence band around anticrossing when magnetic field varies. The $\mathrm{L}$ states in the conduction band show significant tunneling through the barrier between the two rings, while the heavy hole states are mostly localized inside the rings. Furthermore, by comparing Figs. 2a, b, and Fig. 4 we may conclude that the oscillator strength for the transition between the lowest optically active states oscillates, which has been recently confirmed by solving the exciton kinetic equation [21]. Even though we do not take into account the Coulomb interaction between the electron and the hole, we infer that the optical excitonic Aharonov-Bohm effect might be present in the analyzed system.

The oscillator strength for the interband transitions between the electron and the heavy-hole ground states is shown in inset of Fig. 4. We find that the ground exciton state can become completely dark in a narrow range of $B$, where the oscillator strength exhibits minima. These minima take place close to orbital momentum transitions in both the electron and the heavy-hole ground states (see Figs. 2a and 2(b)). These results imply that bright and dark exciton states could alternate in the ground state when magnetic field varies. However, in order to realize such a behavior, the effects of Coulomb interaction should be small. Simple analytical analysis shows that in large rings, where the dimension of the structure is larger than the effective Bohr radius, the motion becomes strongly correlated in a tightly-bound exciton [10]. This is indeed the case in the analyzed structure, where both the rings radii are larger than the effective Bohr radius, which is approximately equal to $12 \mathrm{~nm}$ in GaAs. A rigorous inclusion of the Coulomb interaction demonstrated that the optical excitonic AharonovBohm oscillations are also suppressed in the regime of week interaction [22], which exists in small rings. However, calculation of the exciton states is beyond scope of the present work.

\section{Summary and conclusion}

The effects of a normal magnetic field on the electron and the hole states confined in double concentric type-I nanorings are studied theoretically. The wave functions of the lowest energy electron states are mostly localized inside the outer ring. When the magnetic field is applied, the electron states relocate from the outer ring to the inner ring. Furthermore, anticrossings between states located in different rings appear, which make the Aharonov-Bohm oscillations less regular than in the case of single ring. We showed that the oscillator strength for interband optical transitions between the lowest optically active states varies oscillatory with magnetic field. This effect arises from a strong reduction of the oscillator strength in narrow ranges of magnetic field close to anticrossings between the $l$ states.

\section{Acknowledgments}

This work was supported by the Ministry of Science of Serbia, the EU NoE: SANDiE, the Flemish Science Foundation (FWO-Vl), and the Belgian Science Policy (IAP).

\section{References}

[1] J.M. Garcia, G. Medeiros-Ribeiro, K. Schmidt, T. Ngo, J.L. Feng, A. Lorke, J. Kotthaus, P.M. Petroff, Appl. Phys. Lett. 71, 2014 (1997).

[2] D. Granados, J.M. Garcia, T. Ben, S.I. Molina, Appl. Phys. Lett. 86, 071918 (2005).

[3] M. Tadić, F.M. Peeters, Phys. Rev. B 79, 153305 (2009).

[4] A. Lorke, R.J. Luyken, A.O. Govorov, J.P. Kotthaus, J.M. Garcia, P.M. Petroff, Phys. Rev. Lett. 84, 2223 (2000).

[5] B.C. Lee, O. Voskoboynikov, C.P. Lee, Physica E 24, 87 (2004). 
[6] N.A.J.M. Kleemans, I.M.A. Bominaar-Silkens, V.M. Fomin, V.N. Gladilin, D. Granados, A.G. Taboada, J.M. Garcia, P. Offermans, U. Zeitler, P.C.M. Christianen, J.C. Maan, J.T. Devreese, P.M. Koenraad, Phys. Rev. Lett. 99, 146808 (2007).

[7] R.A. Römer, M.R. Raikh, Phys. Rev. B 62, 7045 (2000).

[8] M. Bayer, M. Korkusinski, P. Hawrylak, T. Gutbrod, M. Michel, A. Forchel, Phys. Rev. Lett. 90, 186801 (2003).

[9] I.L. Kuskovsky, W. MacDonald, A.O. Govorov, L. Mourokh, X. Wei, M.C. Tamargo, M. Tadic, F.M. Peeters, Phys. Rev. B 76, 035342 (2007).

[10] A.O. Govorov, S.E. Ulloa, K. Karrai, R.J. Warburton, Phys. Rev. B 66, 081309 (2002).

[11] T. Mano, T. Kuroda, S. Sanguinetti, T. Ochiai, T. Tateno, J. Kim, T. Noda, M. Kawabe, K. Sakoda, G. Kido, N. Koguchi, Nano Letters 5 No. 3, 425 (2005).

[12] T. Kuroda, T. Mano, T. Ochiai, S. Sanguinetti, K. Sakoda, G. Kido, N. Koguchi, Phys. Rev. B $\mathbf{7 2}$ 205301 (2005).

[13] M. Abbarchi, C.A. Mastrandrea, A. Vinattieri, S. Sanguinetti, T. Mano, T. Kuroda, N. Koguchi, K. Sakoda, M. Gurioli, Phys. Rev. B 79, 085308 (2009).
[14] B. Szafran, F.M. Peeters, Phys. Rev. B 72, 155316 (2005).

[15] B. Szafran, Phys. Rev. B 77, 235314 (2008).

[16] T. Chwiej, B. Szafran, Phys. Rev. B 78, 245306 (2008).

[17] S. Tomić, A.G. Sunderland, I.J. Bush, J. Mater. Chem. 16, 1963 (2006).

[18] O. Stier, M. Grundmann, D. Bimberg, Phys. Rev. B 59, 5688 (1999).

[19] J. Singh, Physics of Semiconductors and Their Heterostructures, Mc Graw Hill, New York 1993.

[20] A. Mühle, W. Wegscheider, R.J. Haug, Appl. Phys. Lett. 91, 133116 (2007).

[21] M. Grochol, F. Große, R. Zimmermann, Phys. Rev. B 74, 115416 (2006).

[22] Z. Barticevic, M. Pacheco, J. Simonin, C.R. Proetto, Phys. Rev. B 73, 165311 (2006). 